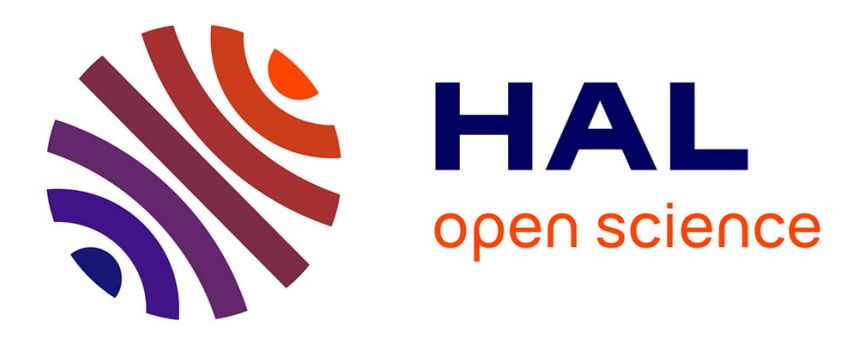

\title{
Deux nouvelles inscriptions de légionnaires de la légion VIII Augusta
}

\author{
Marianne Béraud, Adeline Pichot
}

\section{To cite this version:}

Marianne Béraud, Adeline Pichot. Deux nouvelles inscriptions de légionnaires de la légion VIII Augusta. Archimède: archéologie et histoire ancienne, 2020, Archimède. Archéologie et histoire ancienne, 8, pp.180-194. 10.47245/archimede.0008.ds3.03 . halshs-03280107

\section{HAL Id: halshs-03280107 https://shs.hal.science/halshs-03280107}

Submitted on 7 Jul 2021

HAL is a multi-disciplinary open access archive for the deposit and dissemination of scientific research documents, whether they are published or not. The documents may come from teaching and research institutions in France or abroad, or from public or private research centers.
L'archive ouverte pluridisciplinaire HAL, est destinée au dépôt et à la diffusion de documents scientifiques de niveau recherche, publiés ou non, émanant des établissements d'enseignement et de recherche français ou étrangers, des laboratoires publics ou privés. 
1 DOSSIER THÉMATIQUE 1

SIUE DEUS SIUE DEA. DÉNOMINATIONS DIVINES DANS LES MONDES GREC ET SÉMITIQUE : UNE APPROCHE PAR LE GENRE

98 DOSSIER THÉMATIQUE 2

TRADITION ET TRANSMISSION DANS L'ANTIQUITÉ : RÉFLEXIONS INTERDISCIPLINAIRES

ACTUALITÉ DE LA RECHERCHE

QUOI DE NEUF Á L'OUEST DE STRASBOURG ? KOENIGSHOFFEN :

ÉTAT DES LIEUX ET DÉCOUVERTES RÉCENTES

159 Michel Huмm

Les acteurs de I'archéologie préventive et I'UMR 7044 ArcHiMèdE : une collaboration fructueuse

161 Axelle MURER et Adeline PICHOT

Aux portes de Strasbourg : une occupation de longue durée à Koenigshoffen, en bordure de la route des Romains

180 Marianne BÉRAud et Adeline PICHOT

Deux nouvelles inscriptions de légionnaires de la légion VIII Augusta

195 VARIA 


\section{DEUX NOUVELLES INSCRIPTIONS DE LÉGIONNAIRES DE LA LÉGION VIII AUGUSTA}

\author{
Marianne BÉRAUD \\ Docteur en histoire romaine \\ Professeur d'histoire-géographie
}

marianne.beraud@live.fr
Adeline PICHOT

Docteur ès Lettres Archéologue, Responsable d'opération

ANTEA-Archéologie

adeline.pichot@antea-archeologie.com

\section{RÉSUMÉ}

La fouille archéologique conduite en 2018 au 2 route des Romains, en partie dans la nécropole de Koenigshoffen (Strasbourg), a livré deux nouveaux monuments funéraires. Les inscriptions, datables

\section{MOTS-CLÉS}

Nécropole, monument funéraire, inscription, stèle,

épitaphe,

legio VIII Augusta,

vétéran,

architectus. de l'époque flavienne, sont les épitaphes de légionnaires de la VIIIe légion Auguste. L'un est un ingénieur né à Tolosa ; I'autre, originaire de Vienna, dans la cité des Allobroges, est vétéran. Ces témoignages étoffent le dossier du recrutement provincial dans cette légion.
The archaeological excavation conducted on 2 route des Romains, partly in the necropolis of Koenigshoffen (Strasbourg), produced two new rectangular-based burial monuments. The two gravestones dating from the Flavian period were epitaphs in memory of soldiers belonging to the eighth Legio Augusta. One, born in Tolosa, was an architect; the other, a native of Vienna, was a veteran. These epigraphical remains add new information on the practices of provincial recruitment in this legion.

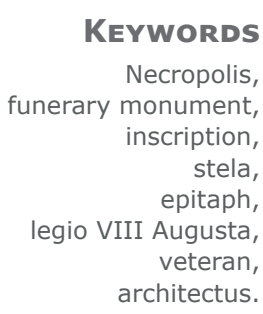


Depuis quelques années, le nombre de trouvailles liées à la nécropole antique de Koenigshoffen s'est étoffé et a permis la mise au jour de pièces majeures, en particulier des stèles funéraires. Ces découvertes sont issues des nombreux chantiers de fouille d'archéologie préventive qui ont eu lieu à la suite des importants travaux d'aménagement, dont la mise en place d'une ligne de tramway, à l'extrémité est du quartier de Koenigshoffen.

La fouille archéologique du 2 route des Romains, située dans l'alignement de I'« allée des tombeaux » découvert en 2015, a permis d'enrichir la collection des monuments funéraires de la nécropole légionnaire de Koenigshoffen de deux inscriptions : celle de Caius Caprius Iulianus et celle de Caius Taedius Secundus, découvertes en juin 2018.

Contrairement à la fouille du 8-20 route des Romains en 2015 , laquelle avait livré des inscriptions de la legio II datées entre 15 et 43 apr. J.-C. [1], les découvertes du printemps 2018 ont mis au jour un nouveau « pôle funéraire ». Ce dernier, chronologiquement plus tardif - fin de l'époque flavienne -, constitue la nécropole de la légion VIII. Ces deux nouvelles épitaphes de légionnaires de la VIIIe légion sont désormais exposées au Palais Rohan (Musée archéologique de Strasbourg).

\section{LA NÉCROPOLE ANTIQUE DE KOENIGSHOFFEN, DES DÉCOUVERTES EXCEPTIONNELLES DEPUIS PLUS D'UN SIÈCLE}

La plus ancienne découverte liée à la nécropole antique remonte à 1851, avec la stèle en calcaire de Lucius Autronius Silo [2], un vétéran de la II légion, originaire de la ville de Norba en Hispanie. Il semble que cette stèle était accompagnée d'une sépulture à crémation encore en place au pied du monument lors de son dégagement. Quelques années plus tard, en 1878, la stèle du soldat Caius Largennius, également légionnaire de la II légion mais provenant de Lucques en Toscane, est dégagée à proximité de la précédente, au sud de la route des Romains. Puis en 1897, environ trente crémations datées du Ire siècle de notre ère apparaissent lors d'une surveillance de travaux. Erwin Kern retrouvera vingt sépultures à crémation dans le même secteur en 1970 . Entre 1884 et 1927, les archéologues dégagent quatre nouveaux monuments (fig. 1) au gré des différents travaux liés au réseau viaire. Il faut ensuite attendre les années 1990 pour découvrir aux 24-26 route des Romains la stèle de Quintus Sertorius Quadratus, légionnaire de la II légion originaire de Vienne [3].

\begin{tabular}{|l|l|l|}
\hline Découverte & Lieu & Année \\
\hline Lucius Autronius Silo & $27-29$ route de Paris & 1851 \\
\hline Titus Julius & 60 rue du Faubourg-Blanc & 1873 \\
\hline Caius Largennius & $57-29$ route de Paris & 1878 \\
\hline Tiberius Babuleius Garrulus & 39 route des Romains & 1884 \\
\hline Fragment de stèle de cavalier & avenue du cimetière & 1908 \\
\hline Titus Albius & 6 route des Romains & 1909 \\
\hline Fragment de stèle & 26 route des Romains & 1927 \\
\hline Quintus Sertorius Quadratus & $22-24$ route des Romains & 1994 \\
\hline $\begin{array}{l}\text { Mausolée de Marcus Valerius Rufus, Lucius Valerius } \\
\text { Niger et Lucius Valerius [...] }\end{array}$ & $9-11$ rue du Donon & 1998 \\
\hline Comnisca & & \\
\hline Publius Modestus Rufrenus & 8 route des Romains & 2012 \\
\hline Quintus Vera[...] Sera[nus] & $8-20$ route des Romains & 2015 \\
\hline Stèle d'un vétéran & $8-20$ route des Romains & 2015 \\
\hline & $8-20$ route des Romains & 2015 \\
\hline
\end{tabular}

Fig. 1. Tableau des stèles découvertes à Koenigshoffen (A. Pichot)

[1] Pour une typologie des stèles funéraires monolithiques découvertes Route des Romains, voir DARDAINE 2017, p. 180 et BLIN 2017, p. 184.
[2] CIL XIII 5975.

[3] $A E 19980981$. 


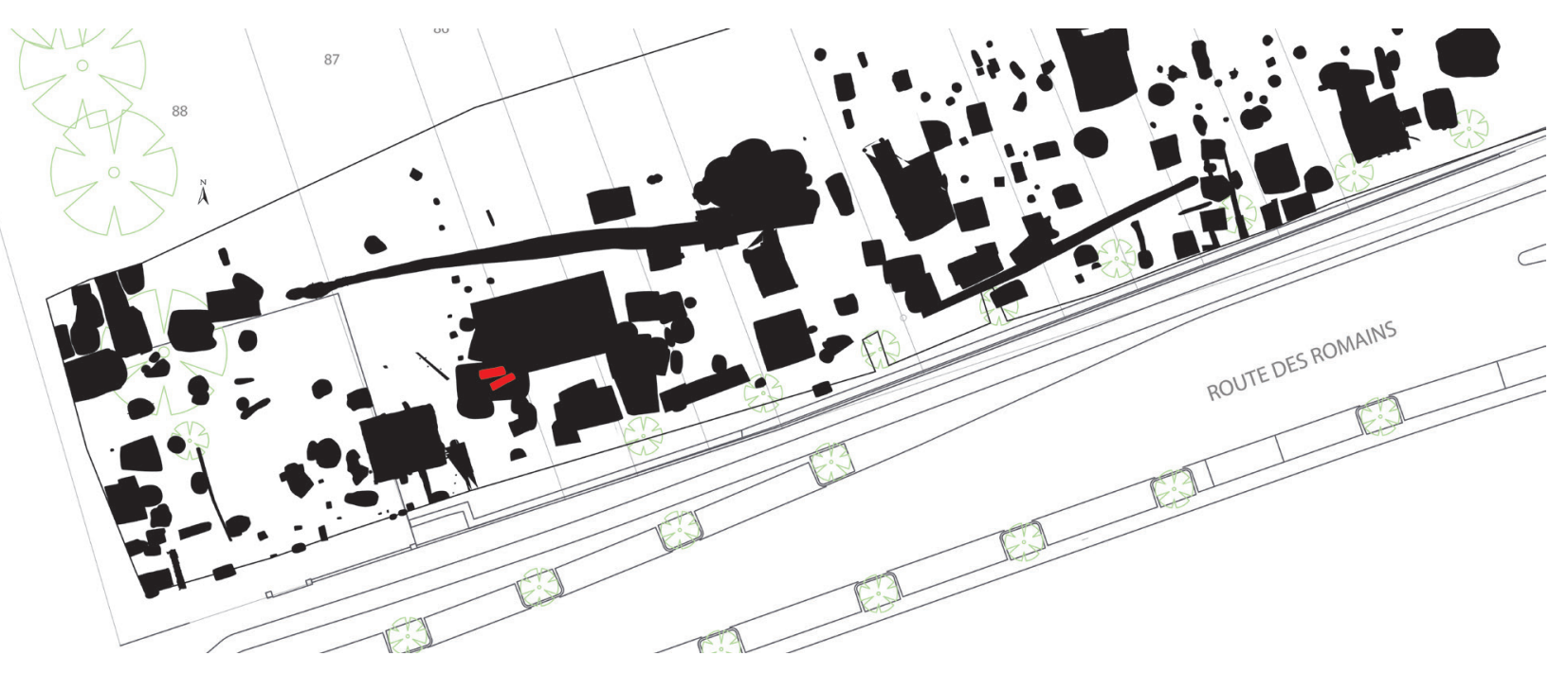

Fig. 2. Carte de situation des stèles sur le chantier au 2 , route des Romains à Koenigshoffen (DAO A. Murer)

La fouille réalisée entre 2014 et 2015 aux 8-20 route des Romains a permis d'apporter des éléments nouveaux [4], mais surtout de restituer la physionomie de la nécropole sur une longueur de 75 mètres, le long de I'emplacement présumé de la voie antique, où dix-huit monuments funéraires et trois nouvelles inscriptions ont été trouvés. Pour la période entre la première moitié du Ier siècle et le début du II ${ }^{\mathrm{e}}$ siècle, une vingtaine de sépultures à crémation et une inhumation ont été étudiées. Toutes les sépultures à crémation sont associées à des tombes individuelles : dans la presque totalité des cas, un seul vase ossuaire a été mis au jour dans les monuments. Dans la première

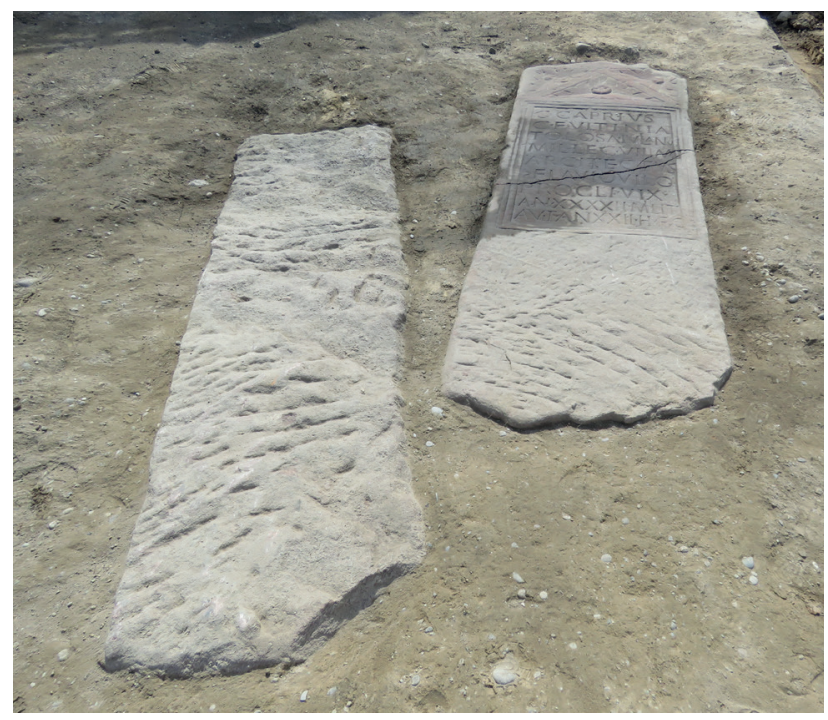

Fig. 3. Disposition des stèles au moment de leur découverte (Cliché A. Pichot) moitié du II ${ }^{\mathrm{e}}$ siècle, les tombeaux de la nécropole sont démantelés et remblayés. Les fondations ont néanmoins été majoritairement préservées, quelques enclos conservant des parties en élévation. Une partie des blocs d'architecture des tombeaux a été poussée à l'arrière de ces derniers, dans des fosses creusées à cet effet, ce qui incite à restituer un chantier de démantèlement coordonné. Ce n'est qu'après cette entreprise qu'un remblaiement et un nivellement du terrain sont effectués afin d'y installer des parcelles d'habitation [5].

\section{CONTEXTE ARCHÉOLOGIQUE DES STĖLES DU 2, ROUTE DES ROMAINS}

Les stèles de Caius Caprius Iulianus et de Caius Taedius Secundus ont été découvertes au mois de juin 2018 [6]. Elles étaient déposées à plat, au fond d'une fosse rectangulaire, à une dizaine de mètres de la limite sud du chantier (fig. 2 et $\mathbf{3}$ ). La première stèle découverte, celle de Caius Caprius Iulianus, présentait sa face épigraphique vers le haut, orientée est-ouest. La seconde était posée face épigraphique contre terre dans un axe sud-ouest - nord-est. La datation de leur dépôt dans cette fosse a été rendue possible grâce à l'identification d'un reste d'une semelle de chaussure, situé entre les deux stèles à environ 20 $\mathrm{cm}$ de profondeur sous leur niveau d'apparition. Les clous de la chaussure permettent de la dater entre

[4] BLIN \& FLOTTÉ 2017, p. 174-180.

[5] BLIN \& FLOTTÉ 2017, p. 178.

[6] Murer et al. 2020. 
le XIXe et le début du XXe siècle [7]. La présence de mobilier antique et médiéval est certainement liée au creusement de cette fosse dans le fond de cabane 563, lui-même installé sur les fosses romaines 143 et 141 .

Nous supposons donc que les stèles ont été couchées une première fois lors du démantèlement de la nécropole, afin de laisser place aux premiers bâtiments entre les années 120 et 180 de notre ère. Elles ont pu, comme aux 8-20 route des Romains, être laissées in situ ou juste à côté de leur emplacement d'origine, puis redécouvertes à l'époque contemporaine pour être enfouies de nouveau, certainement pendant l'annexion allemande (1871-1918), lors du réaménagement du quartier. Elles ont probablement été jetées sans ménagement dans une fosse, la plus grande stèle présentait d'ailleurs, dès sa découverte, une fissure montante en son centre sur pratiquement toute sa largeur, au milieu du champ épigraphique. Il est étonnant que cette découverte n'ait pas été recensée par les érudits de l'époque, alors que d'autres stèles exhumées pendant la seconde moitié du XIX siècle ont été inventoriées.

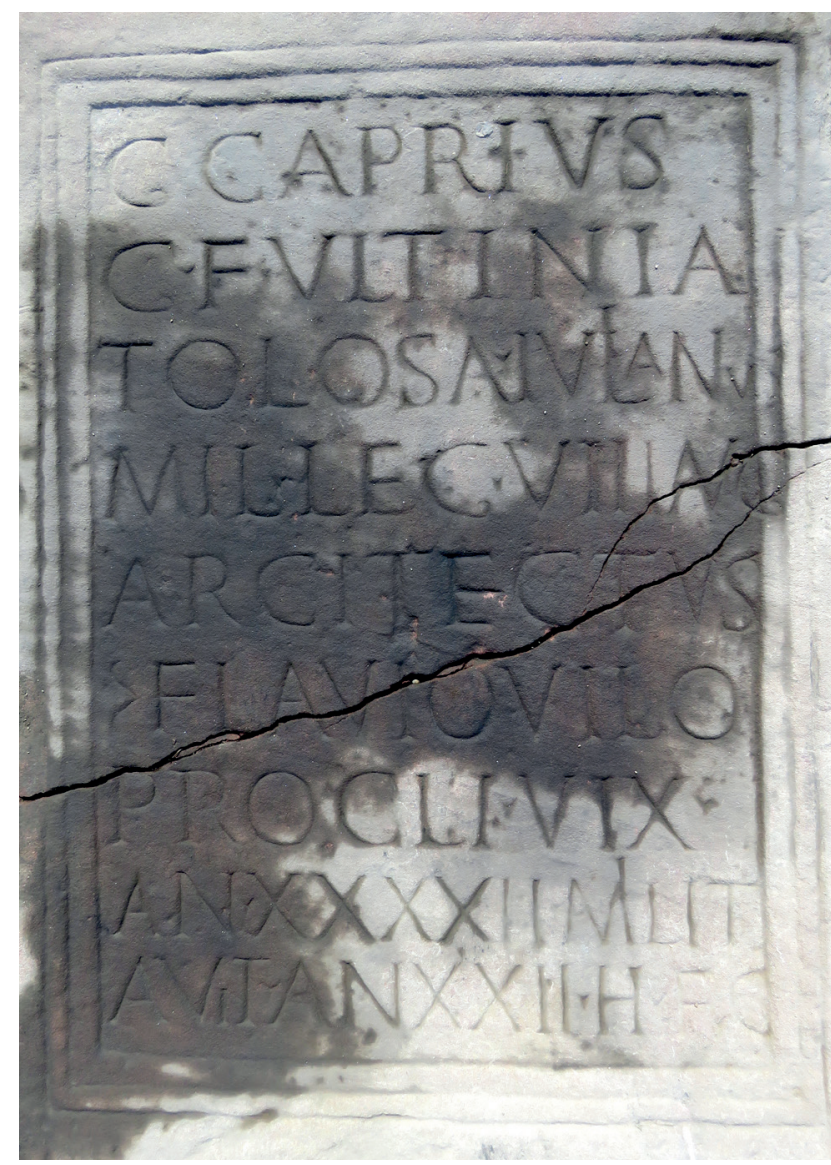

Fig. 4. In situ, découverte de l'épitaphe de C. Caprius Iulianus (Cliché A. Pichot)
Les stèles ont été restaurées par le musée de Strasbourg, où elles sont actuellement exposées. Elles sont toutes les deux taillées dans un bloc monolithe de grès probablement vosgien, la qualité de la pierre étant très différente d'une stèle à l'autre. Ce matériau est employé globalement au II siècle à Koenigshoffen dans des contextes d'habitat, de constructions publiques [8] et pour les stèles funéraires dès la fin du Ier siècle de notre ère [9].

\section{LE MONUMENT DE C. CAPRIUS IULIANUS.}

La stèle de C. Caprius Iulianus est l'épitaphe d'un soldat du génie de la legio VIIIa Augusta, ingénieur, à Argentoratum. Elle fut découverte couchée face inscrite vers le haut, complète mais cassée en deux fragments jointifs (fig. 4).

Ce monument funéraire parallélépipédique rectangle mesure $209 \mathrm{~cm}$ de long, pour une largeur de $74 \mathrm{~cm}$ et une épaisseur de $21,5 \mathrm{~cm}$; il est taillé dans un grès gris au grain fin. Le champ épigraphique et le fronton sont en bon état de conservation, nous avons pu immédiatement après le dégagement sur le terrain lire l'inscription épigraphique.

L'épitaphe occupe la moitié supérieure de la stèle, elle prend place dans un cadre mouluré qui est surmonté par un fronton ouvragé de trois zones triangulaires séparées par des bandeaux (fig. 5). Sur le tympan, une rosette à quatre pétales est placée au milieu d'un feuillage, quelques traces de piquetage sont visibles dans le creux des feuilles. Dans les écoinçons, un simple point orne les feuillages, celui de gauche est assez abîmé. Le sommet de la stèle est légèrement triangulaire et plusieurs épaufrures sont visibles en partie droite.

La partie basse de la stèle est grossièrement taillée au pic (fig. 6), comme les tranches et la face non épigraphique. Une zone d'environ $40 \mathrm{~cm}$ de haut, située sous le registre épigraphique, a été ravalée plus finement au ciseau ; elle devait être visible lorsque la stèle était en place dans le sol. La partie fichée en terre n'est pas complète, en effet elle mesure à peine $38 \mathrm{~cm}$ de haut, avec un bord inférieur très abîmé, ce qui est insuffisant pour assurer une bonne stabilité à la stèle une fois mise en place. Cette extrémité a pu être cassée lors

[7] Selon I'expertise de Marquita Volken de Gentle Craft (Lausanne).

[8] Hormis nos découvertes, parmi les 34 stèles trouvées à Strasbourg, 26 sont en grès et huit en calcaire; voir HARTKOPF-FRÖDER \& JODRY 2016, p. 343.

[9] BLIN \& FLotTÉ 2017, p. 192. 


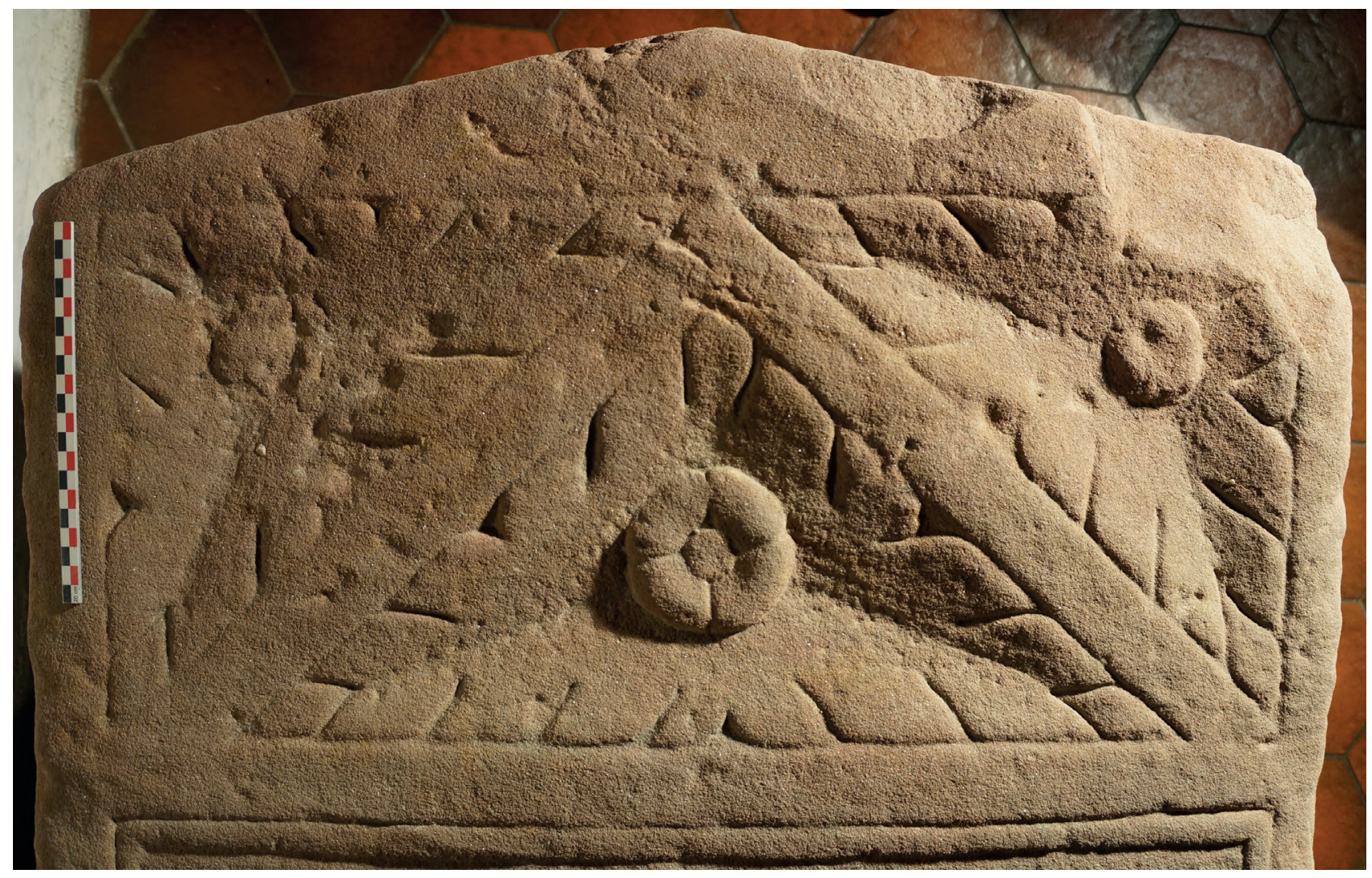

Fig. 5. Détail du fronton après nettoyage (Cliché A. Pichot)

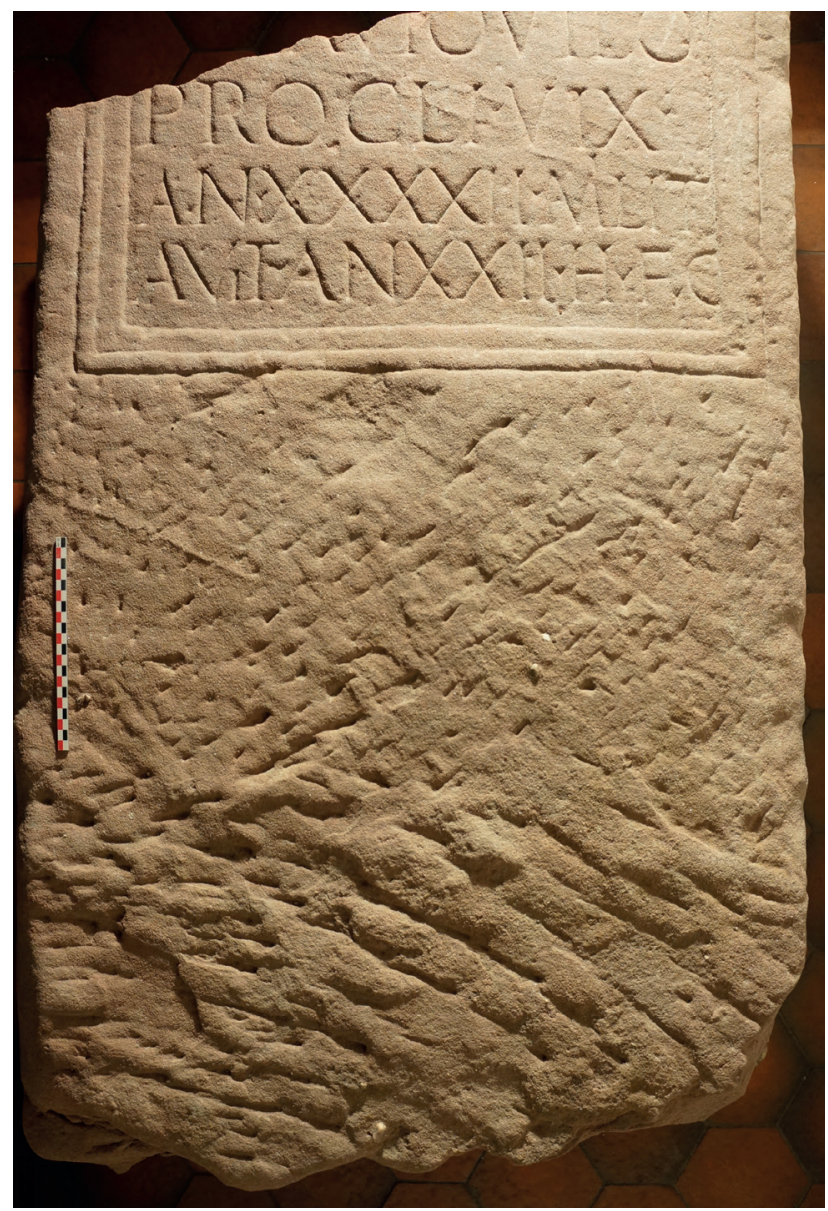

Fig. 6. Détail de la partie basse après nettoyage (Cliché A. Pichot)

du démantèlement de la nécropole. Deux possibilités s'offrent à nous : soit il manque une cinquantaine de centimètres de long à la stèle, ce qui permet d'obtenir $1 / 3$ de la hauteur totale de la stèle plantée en terre ; soit son extrémité était constituée d'un large tenon parallélépipédique qui prenait place dans une mortaise constituée d'un socle en grès, comme cela a été vu aux 8-20 route des Romains [10].

La forme du monument (stèle à sommet plat avec tympan triangulaire) est similaire à celle d'autres épitaphes de légionnaires, découvertes dans la région rhénane, à Strasbourg : stèle de T. Flavius Peregrinus (AE 2010 1067), monument de C. Largennius (CIL XIII 5978) ou à Mayence : épitaphes de Petronius (CIL XIII 6871) et de P. Solius Suavis (CIL XIII 6972). Le champ épigraphique $(82 \mathrm{~cm}$ sur $57 \mathrm{~cm})$, abaissé par un surcreusement, est cerné par une double mouluration du cadre. Si le bord inférieur du cadre est

[10] BLIN \& FLOTTÉ 2017, p. 187. 
parfaitement horizontal, le bord supérieur dévie à son extrémité droite vers le bas, mais l'ensemble des lignes du texte est parfaitement aligné (fig. 7). L'ordinatio soignée dénote le travail d'un lapicide rompu à son art : le texte, composé de neuf lignes, est proprement aligné à gauche, avec des interlignes de $2 \mathrm{~cm}$ et plusieurs interponctions triangulaires (I. 1, 2, 3, 4, 7, 8, 9).

Les lignes sont régulières et le module des lettres, d'une belle graphie en écriture quadrata, oscille entre $5,8 \mathrm{~cm}$ et $3,5 \mathrm{~cm}$ (pour les lettres naines, par ex. A I. 3 de Iulianus). Lettres finement pattées. L. 3 : en fin de ligne, $\mathrm{S}$ sur le cadre ; I. 4 : dernière lettre $\mathrm{G}$ sur le retour de moulure. L. 1 et 2 : La lettre avant Caprius (I. 1 ) et devant $f$ (ilius) est un $\mathrm{G}$ et non un $\mathrm{C}$ : la boucle du $\mathrm{G}$ est strictement identique à celle du G de LEG (I. 4). Ligatures : I. 3 : ligature du L et du I (I long) : I dans le prolongement du L ; I. 4 : AV ; I. 8 : du M et du I. Lettres naines : I. 3 : A, entre LI et $N$; $V$ et $S$, après $\mathrm{N}$ de Iulianus et I. 9 : I. L. 6 : glyphe rétroverse pour centuria. La grande cassure oblique traverse les lignes 5 et 6 . Coupures non syllabiques des mots en fin de ligne.

L'établissement du texte est le suivant (fig. 7) :

G CAPRIVS
G F VLTINIA
TOLOSA IVLIANVS
MIL LEG VIII AVG
ARCITECTVS
$>$ FLAVIOVILO
PROCLI VIX
AN XXXXII MILIT
AVIT AN XXII H F C

G(aius) Caprius / G(ai) f(ilius) V<o>ltinia / Tolosa Iulianus / mil(es) leg(ionis) VIII Aug(ustae) / $\operatorname{arc}(h)$ itectus / (centuria) Flauio Vilo / Procli uix(it) / an(nos) XXXXII milit/auit an(nos) XXII; h(eres) f(aciendum) $c$ (urauit).

G. Caprius Iulianus, fils de Gaius, de la tribu Voltinia, originaire de Tolosa, soldat de la huitième légion Auguste, ingénieur dans la centurie de Proclus, sous le commandement de Flavius Vilus. Il vécut 42 ans, servit dans l'armée pendant 22 ans. Son héritier a pris soin de faire (ce monument).

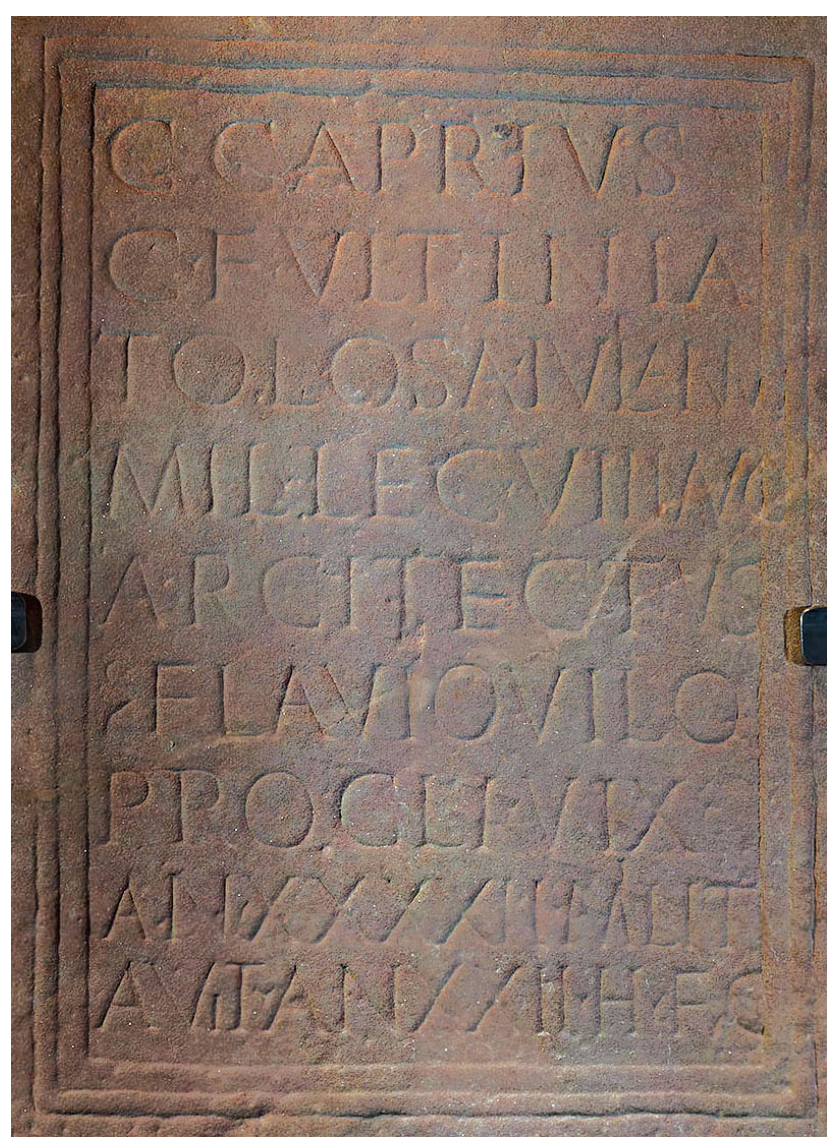

Fig. 7. Détail du titulus après restauration (Cliché $A$. Touzet)

Le formulaire initial au nominatif, I'absence de mention aux dieux Mânes, I'installation de la legio VIIIa à Argentoratum (ca 90), le contexte archéologique, plaident en faveur d'une datation de la fin du Ier siècle apr. J.-C.

Le dédicant, anonyme, est I'héritier du dédicataire, G. Caprius Iulianus, Tolosate d'origine, issu des Volques Tectosages. La déclinaison de l'identité du défunt prend la forme d'une dénomination latine complète du citoyen romain (tria nomina, filiation, tribu, origine). Elle fut probablement copiée sur le matricule de son « livret militaire ».

Le gentilice italien Caprius, nom qui évoque pour I. Kajanto la faune caprine [11], concentre ses occurrences dans deux régions : le Samnium et le Latium. Dans le Samnium, il est répandu autour de Corfinium/ Corfinio, et dans les collines des Abruzzes (6 fois) (Forum Nouum/Vescovio et Furfo/Barisciano) [12].
[11] KAJANTO 1965, p. 326.

[12] Corfinio : CIL IX 3171 (Capria Quinta); Vescovio : CIL IX 4807 (L. Caprius Hierax) ; Barisciano : CIL IX 3525
(Q. Caprius Optatus), 3539 (Q. Caprius Ferox) ; Ofena : CIL IX 3391 (A. Caprius) ; Chieti : AE 1980368 (Caprius Charitinus). 
Dans le Latium (12 fois), il est bien attesté, en particulier à Aquinum, actuelle Aquino [13]. Rare dans les Gaules, ce gentilice est porté deux fois en Gaule Narbonnaise : à Narbonne (A. Caprius : AE 1998 918) et à Ernaginum/St Gabriel (Q. Caprius Hermes : CIL XII 987), près de Tarascon. C'est sa première attestation dans la cité de Toulouse. Il est aussi mentionné dans une inscription d'Aquitaine, à Saintes, où il est porté au I $^{\text {er }}$ siècle par un citoyen romain de première génération M. Caprius Secundus, fils de Comnertus (CIL XIII 1061) [14]. Le cognomen Iulianus est très fréquent, notamment en Narbonnaise où on en recense plus de 45 occurrences [15], presque toutes au masculin. Sa filiation indique en effet qu'il n'est pas un citoyen de première génération. Toutefois, la rareté de son nomen et le fait qu'il soit un unicum dans la cité de Toulouse plaide en faveur de l'octroi récent de la ciuitas à sa famille, soit par son père soit par son grand-père.

Originaire de Toulouse, G. Caprius Iulianus est inscrit dans la tribu Voltinia, attribuée aux Volques Tectosages par Auguste. En 27 av. J.-C. [16], année du voyage d'Auguste en Transalpine au cours duquel il réorganise la province qui devient alors Narbonnaise, la cité de Tolosa reçut le ius Latii, et devint colonie latine. Pour J.-M. Pailler, Domitien aurait ensuite érigé Toulouse au rang de colonie romaine, en lui octroyant le titre de Palladia [17]. Dans tous les cas, la séquence onomastique de ce Tolosate dénote une ambiance culturelle bien ancrée dans la romanité.

Le parcours militaire de la VIII légion Auguste, étudié par M. Reddé, révèle qu'elle fut d'abord cantonnée sur le Danube inférieur, à Nouae (Bulgarie), où elle s'était installée en 45 apr. J.-C. pour participer à la guerre de Didius Gallus contre Mithridate, roi du Bosphore [18]. Ce corps de troupe fait son entrée en Gaule en 70, lorsque Vespasien l'intègre dans le dispositif militaire

[13] A Aquino : CIL X 5459 (Cn. Caprius), 5460 (C. Caprius Chilo et C. Caprius Stephanus), 5461 (L Caprius Rufus). Le nom apparaît aussi chez Horace, Satires, livre 1, satire 4, 66 et 70. C'est celui d'un accusateur de métier redouté. [14] ILA Santons, 27. Cela montre I'affinité phonique qu'a pu revêtir ce gentilice italien pour un indigène - avec le gaulois gabro, la chèvre, - qui fut le premier de sa famille à devenir un citoyen romain.

[15] LörINCZ 1999, s.v. Iulianus.

[16] Provost \& Pailler 2017, p. 102.

[17] PAILLER 1988, p. 107. Pour honorer peut-être un compagnonnage ancien avec le plus illustre des toulousains, M. Antonius Primus, vainqueur de Vitellius et maître de Rome en 69, puis mis à l'écart par Vespasien et par Mucien. [18] Tacite, Annales, XII, 15. A ce sujet, voir REDDÉ 2000, p. 124.

[19] Sur la durée du service légionnaire (25 ans ? 20 ans ?), question débattue, voir SPEIDEL 2009, p. 329 et MANN 2000, p. 155. Certains historiens admettent que la création par Tibère de la garde prétorienne réforma le décret augustéen de renforts pour mater la révolte des Lingons. C'est alors le camp de Mirebeau qui accueille la VIII Augusta avant son transfert et son installation définitive à Argentoratum (Strasbourg).

Mort à Strasbourg à 42 ans et ayant accompli 22 ans de militia, G. Caprius Iulianus s'est engagé dans la légion VIII Augusta à 20 ans. Cette inscription montre qu'à l'époque flavienne, on peut mourir miles en ayant accompli plus de vingt ans de service. Cela conduit à réinterroger la durée pendant laquelle les légionnaires devaient servir sous les enseignes avant de recevoir I'honesta missio [19]. Désigné comme simple miles, Iulianus n'avait donc pas, au moment de sa mort, achevé le temps réglementaire de mobilisation.

La trajectoire de ce Tolosate est étroitement liée à la «Guerre Civile » de 68-69 et à I'« année des quatre empereurs »[20]. Le service militaire de G. Caprius Iulianus a probablement commencé à Mirebellum/ Mirebeau, en Côte-d'Or (vallée de la Bèze, à 25 km de I'actuelle Dijon), où la legio VIII stationna à la suite du soulèvement des Lingons contre la politique cadastrale de Galba [21], puis contre Vespasien. Ardent partisan de Vitellius [22], le peuple lingon s'opposa après la bataille de Crémone, en 69 apr. J.-C., à Vespasien, sous I'autorité de son chef, Iulius Sabinus - à la tête d'une armée de 6000 hommes -, probablement un aristocrate dont un ancêtre avait été fait chevalier par César (Tacite, Hist. IV, 55). Vespasien retira la citoyenneté romaine aux Lingons et les plaça sous la surveillance directe de la legio VIII qu'il installa, sous l'autorité de Frontin, à Mirebeau [23]. Il est fort plausible que G. Caprius Iulianus fut enrôlé à la faveur de ce soulèvement des Lingons qui engendra une militarisation du secteur. Aussi participa-t-il, selon toute vraisemblance, aux campagnes de Domitien contre les Chattes en Vettéravie en 88-89 apr. J.-C. et à la répression de

de 13 av. J.-C. sur le temps de service (16 ans pour les légionnaires et 20 ans pour les auxiliaires) : désormais Tibère impose un service de16 ans pour les prétoriens, 20 ans pour les légionnaires et 25 ans pour les auxiliaires. [20] Cosme 2012, p. 34.

[21] Les Lingons sont les victimes de la politique de Galba qui ampute leur territoire, alors que les Séquanes et les Eduens, dont I'intégrité du territoire est préservée, ont obtenu une remise de leur tribu et des libertés impériales (Tacite, Histoires, I, 51). Sur le camp légionnaire de Mirebeau et I'installation de la légion à Strasbourg, voir Goguey \& REDDE, 1995, p. 379 qui remet en cause l'hypothèse d'une installation de la légion VIII à Strasbourg au début des années 70 reposant sur des fouilles stratigraphiquement datées et la lecture lacunaire du fameux légionnaire d'Offenburg. Voir aussi Redde 1993, p. 48, REDde 1996a, p. 192 ; RedDE 2006, p. 331 ; Goguey 2008, p. 230.

[22] Tacite, Histoires, I, 57, 64 et 78 : Othon leur octroie la citoyenneté romaine.

[23] Frontin, Stratagèmes, IV, 3, 13. 
la révolte du légat Saturninus à Mayence [24]. Vers 93-97 apr. J.-C., G. Caprius suivit sa légion, transférée sur le limes rhénan, à Argentoratum/Strasbourg [25], pour contrôler un territoire militaire et administratif situé entre la frontière de Rhétie et le bas Neckar. Une fois la Lingonie pacifiée, la présence de la VIII légion à Mirebeau n'était plus nécessaire. En revanche, I'organisation du limes du Neckar, la liaison entre la région du Main inférieur et le Danube nécessitait une installation de la légion plus près de la frontière, à Strasbourg. L'épitaphe mentionne la profession du défunt qui appartient au corps des soldats du génie de la huitième légion : C. Caprius Iulianus était architectus. En nous fondant sur l'étude de C. Schmidt Heidenreich sur les légionnaires bâtisseurs, nous pouvons penser que l'ingénieur [26] Iulianus fait partie de la liste des immunes - les exemptés de corvées -, à l'instar de l'arpenteur (mensor) et du géomètre-niveleur (librator) [27]. Son métier consiste à planifier la disposition des édifices internes du camp : principia, praetorium, bains, baraquements [28]. L'ingénieur régit la construction de ponts, de murs de circonvallation, I'élaboration des machines de guerre ou de siège, la construction des camps permanents (castra statiua $=$ dits " camps d'hiver $\gg$ ) ou des camps de marche (castra aestitua = « camps d'été »).

G. Caprius Iulianus opère sous le commandement de Flavius Vilus [29], le centurion d'une unité, une centurie, qui porte le nom de son fondateur et premier centurion, Proclus. Dans l'épigraphie, une centuria Procli est attestée en Dalmatie, mentionnée dans une inscription d'Argyruntum/Starigrad [30], près de Burnum. Or, après son ralliement à Vespasien, victorieux de la bataille de Crémone, la huitième légion fit un bref séjour à Burnum [31]. Ce faisceau d'indices [32] laisse penser qu'il s'agit bien de la centurie de Proclus sous les fanions de laquelle fut enrôlé G. Caprius Iulianus. Il s'agit donc de la seconde attestation de cette unité de la huitième légion [33].

Du point de vue de l'organisation du génie militaire, cette inscription présente l'intérêt d'associer un architectus à un type d'unité, la centurie. Jusque-là, seul un ex-voto de Mayence témoignait de la présence d'un architectus au moins par centurie : celui d'Aelius Verinus, ingénieur dans la centurie de Nigidius Censorinius à Mogontiacum/Mayence (CIL XIII 6680). À Strasbourg, trois autres légionnaires de la VIII ${ }^{e}$ légion sont connus : L. Licinius Maximus Aequo, de la tribu Claudia (CIL XIII 5982), T. Flavius Peregrinus, originaire de Milan (CIL XIII 5979) et C. Tadeius Secundus, Allobroge de Vienne, vétéran (inédite, voir infra).

Tout comme ce dernier, G. Caprius Iulianus appartient à une période charnière en termes de recrutement militaire, la fin du règne de Domitien. Ainsi que l'ont démontré G. Forni et J. C. Mann, à partir du règne de Vespasien, la proportion d'Italiens dans les légions diminua. La fin du Ier siècle vit l'extinction progressive du recrutement italien [34]. Les citoyens de la péninsule furent remplacés par les provinciaux les plus romanisés, originaire de Narbonnaise ou d'Hispanie. En effet, les provinciaux, qui représentaient le tiers des recrues à l'époque de Tibère, fournissent la moitié des effectifs sous le règne de Néron, et plus des trois quarts à l'époque flavienne. Par ailleurs, les comptages réalisés par $D$. et $F$. Tassaux sur le recrutement des soldats originaires des Gaules ont permis de démontrer que, au Ier siècle, la Gaule Narbonnaise a fourni 270 recrues (35\% des soldats originaires de l'ensemble des Gaules
[24] GogueY \& REDDÉ, 1995, p. 379 à partir des sondages menés à Mirebeau, sur l'emplacement des anciennes usines Istra, précisent que la VIIIe légion quitta définitivement Mirebeau après la révolte de Saturninus.

[25] REDDÉ 2000, p. 123 : la date précise de cette installation à Argentorate n'est pas connue, peu après 90 . Voir aussi ReDDÉ 1996b, p. 204 ; RedDÉ 1997, p. 9 et RedDÉ 2010, p. 29-32 qu propose une fourchette chronologique entre 93 et $97 \mathrm{apr}$. J.-C. [26] FLEURY, 2011 démontre qu'architectus doit être traduit plutôt par « ingénieur » que par « architecte ».

[27] SChMidt HEIDENREICH 2012, p. 335, l'architectus exerce un métier technique de la construction dans le camp qui fait de lui un technicien spécialisé, à l'inverse des métiers manuels comme ceux du charpentier (tignarius) ou du stucateur (tector).

[28] Sur les architecti, voir DoNDERER, 1996 et BARDOUILLE 2010, p. 83 ; STOLL 1998, p. 220 et, pour le monde civil, KoLB 2008, p. 102.

[29] Il n'y a certes pas d'interponction entre Flavius et Vilus, mais il convient de noter que le lapicide, bien que très soigneux, n'a pas inséré non plus d'interpunctio entre le $\mathrm{G}$ et le $\mathrm{C}$ de
Caprius (I.1), ni entre AN et XII (I. 9). Par ailleurs, si Flavius est connu, Vilus est possible. En revanche, nous ne disposons d'aucune attestation du nom Flaviovilus. On ne peut donc lire autrement que Flavius Vilus.

[30] ILJug. III 2896 (Argyruntum) : L(uci) Valeri / (centuria) Pocli.

[31] REDDE 2000, p. 122 à partir des briques de la huitième légion à Burnum (Dalmatie). Voir aussi, à Burnum, l'épitaphe du Viennois S. Valerius Lucinus, soldat de la VIII legio Augusta, légionnaire dans la centurie de Terentius Iulianus (ILJug. III 2818)

[32] Sur les centurions légionnaires, voir FAURE 2013.

[33] On sait par ailleurs qu'un Larginius Proclus, vivant en Germanie au Ier siècle, aurait prédit à Domitien sa mort. Pour cette prédiction funeste, il fut condamné à mort mais parvint à échapper à son exécution (Dion Cassius, Histoire romaine, 68, 16 et Suétone, Vie de Domitien, 16). Aucun indice ne permet d'étayer rigoureusement la thèse rapprochant ce personnage du Proclus de notre inscription.

[34] FORNI 1953, p. 180, appendice 2 ; FORNI 1974, p. 356 et MANN 1983. 


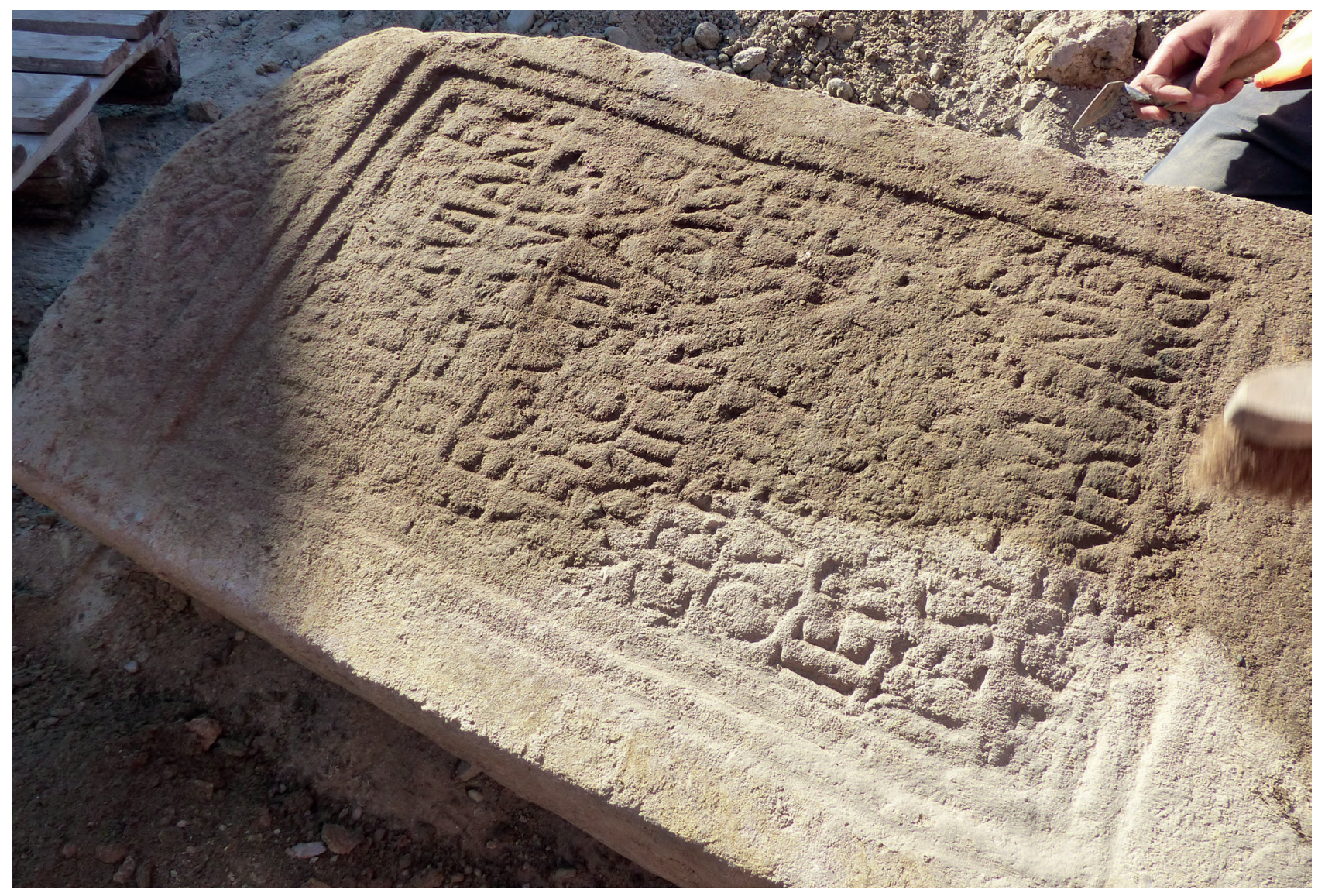

Fig. 8. La stèle de C. Taedius Secundus au moment de la dépose (Cliché M.-L. Bonte)

et Germanie), devant la Gaule Lyonnaise (52 recrues) et la Germanie Supérieure (110 recrues) [35]. La présence de G. Caprius Iulianus, Tolosate, à Argentoratum sous le règne de Domitien s'inscrit dans cette double tendance : recul des Italiens et forte participation des natifs de Narbonnaise dans les légions romaines.

Toutefois, l'intégration dans cette tendance ne doit pas occulter que, dans la région rhénane, comme dans les Gaules en général, les Volques Tectosages participèrent très faiblement à la conscription volontaire et au dilectus. Au total, on ne connaît que dix Tolosates ayant intégré l'armée romaine, principalement en Pannonie et en Germanie inférieure. Sur le front du Rhin, deux inscriptions de Mogontiacum/Mayence mentionnent des Volques Tectosages de Toulouse dans les légions rhénanes : C. Iulius Priscus (CIL XIII 6867) dans la légion III Macedonica, et Q. Octavius (CIL XIII 6904) dans la légion XIIII Gemina. À l'évidence, la cité de Toulouse ne fait pas partie de ces ciuitates de Gaule Narbonnaise (Vienne en premier lieu, mais aussi Vaison-la-Romaine, Nîmes et Arles) dont des citoyens ont intégré les contingents de l'armée romaine dans les Gaules et les Germanies. Ce nouveau témoignage d'un Tolosate dans l'armée enrichit donc considérablement le corpus sur les Tectosages hors de Toulouse.
Le nom du dédicant est inconnu : cela laisse présumer que la stèle fut commandée par un frère d'arme de G. Caprius Iulianus.

\section{LA STÈLE DE C. TAEDIUS SECUNDUS.}

Cette épitaphe est celle d'un Allobroge, C. Taedius Secundus, originaire de Vienne (Isère), vétéran de la VIII Augusta à Argentoratum.

D'un module plus petit que la précédente, cette stèle parallélépipédique à sommet plat mesure $213 \mathrm{~cm}$ de long pour une largeur de $58 \mathrm{~cm}$ et une épaisseur de $21 \mathrm{~cm}$; elle est taillée dans un grès rouge grossier avec de nombreuses inclusions. Si la stèle est complète, le champ épigraphique et le fronton sont en mauvais état de conservation, cela est peut-être dû à un matériau de moins bonne qualité et à un dépôt face épigraphique contre terre dans la fosse (fig. 8).

[35] TASSAUx 1996, p. 196. À partir des Antonins et des Sévères, le recrutement se fait essentiellement dans les provinces du nord-est de la Gaule, et non plus en Narbonnaise, les provinces de Lyonnaise et d'Aquitaine n'ayant jamais occupé une place importante. 


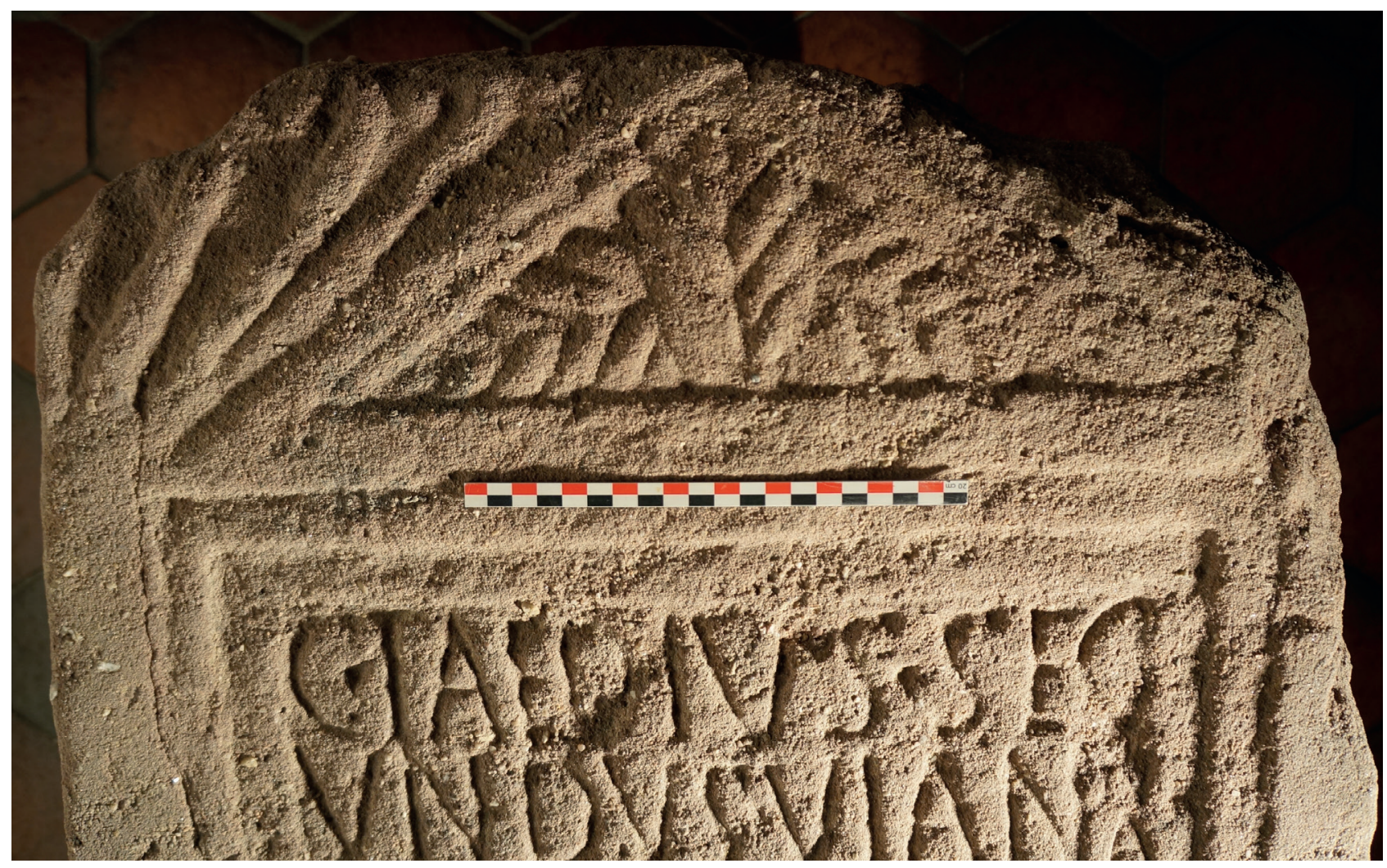

Fig. 9. Détail du fronton après nettoyage (Cliché A. Pichot)

Les registres sont disposés de la même façon : une épitaphe sur la moitié supérieure de la stèle prenant place dans un cadre mouluré, surmontée par un fronton quadrangulaire décoré (fig. 9). Au centre de ce dernier, le tympan est orné d'un rameau dressé, entouré de chaque côté par un rameau tombant qui épouse les angles du triangle. L'extrémité droite de la stèle est manquante et si celle de gauche est abîmée, il est possible de distinguer un motif de stries évoquant des branches.

Ce bloc était enfoncé en terre sur une hauteur de $70 \mathrm{~cm}$, environ $1 / 3$ de la hauteur totale. Le traitement de l'épiderme le confirme puisque le bas de la stèle est grossièrement taillé au pic et la partie médiane travaillée au ciseau sur $31 \mathrm{~cm}$ de hauteur, avec une bande de $10 \mathrm{~cm}$ de large en partie gauche polie (fig. 10).

Le champ épigraphique $(74 \mathrm{~cm}$ sur $47 \mathrm{~cm})$, surcreusé, est cerné par une double mouluration. Le texte, composé de douze lignes, est grossièrement justifié, de sorte que l'ordinatio est médiocre. Nous remarquons plusieurs interponctions triangulaires et des traces de réglure.

La taille des lettres est comprise entre $5 \mathrm{~cm}$ (1 1 ère ligne), $4 \mathrm{~cm}$ (I. 12) et $3 \mathrm{~cm}$ pour les lettres naines (A de Viana, I. 2). L. 2 : A sur la moulure. L. 3 : T long (littera columnata), lié aux E. L. 4 : tilde sur le VIII.
L. 6 : L sur le contour du cadre et I. 9, 10, 11 : dernières lettres $X, O, V$ sur le retour de moulure. L. 12 : RAVIT déborde sur la mouluration. Barre horizontale des T sinusoïdale. Interligne : $1 \mathrm{~cm}$.

\section{TAEDIVS SEC \\ VNDVS VIANA \\ VETERANVS \\ LEG VIII AVG \\ AERORVM XXV \\ VIXIT ANNOS L \\ C BRINNVS \\ QVINTVS VE \\ TER HERES EX \\ TESTAMENTO \\ FACIENDVM CV \\ RAVIT}

C(aius) Taedius Sec/undus / Vian(n)a / ueteranus / legio(nis) VIII Aug(ustae) / aerorum XXV / uixit annos $L / C$ (aius) Brinn(i)us Quintus ue/ter(anus) heres ex / testamento / faciendum cu/rauit.

C. Taedius Secundus, originaire de Vienne, vétéran de la huitième légion Augusta, a servi 25 ans, a vécu cinquante ans. C. Brinnius Quintus, vétéran, a pris soin de faire, conformément au testament. 


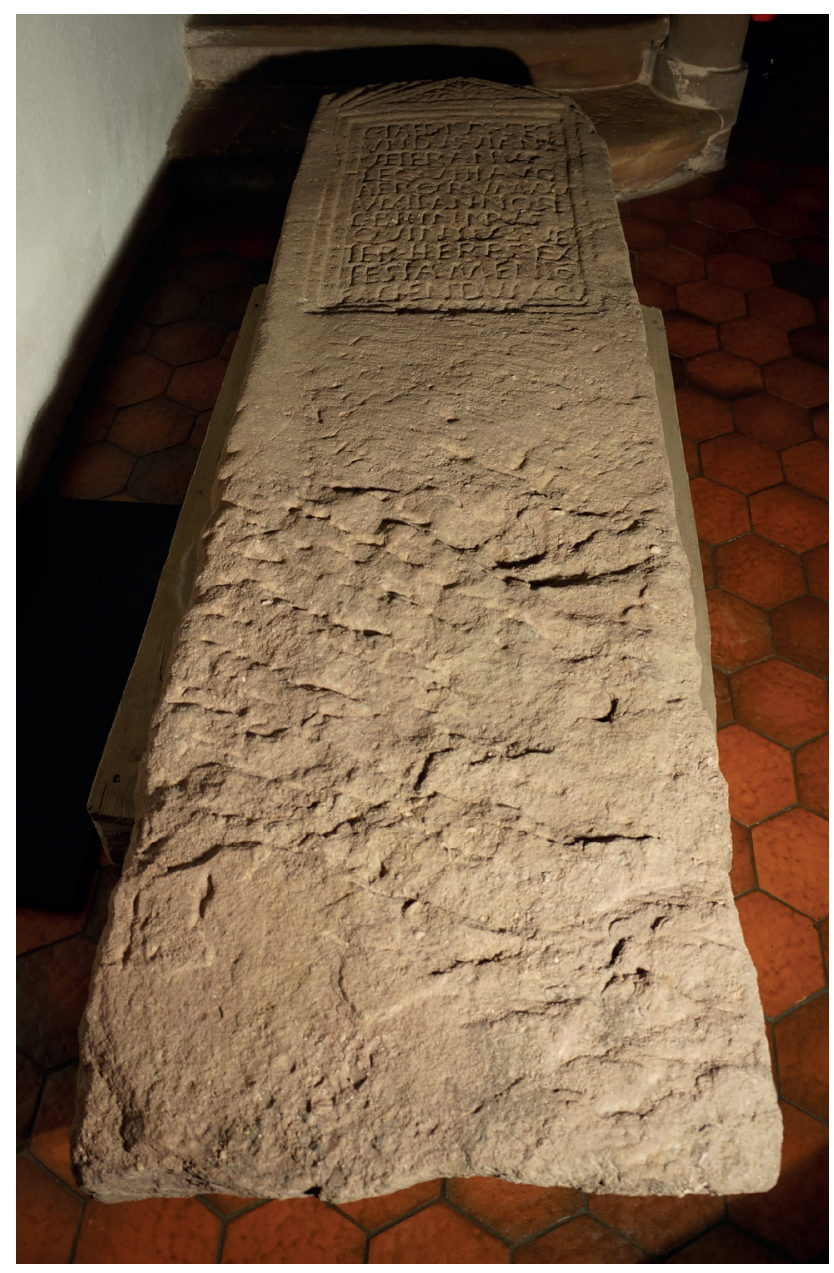

Fig. 10. Détail de la partie basse après nettoyage (Cliché $A$. Pichot)

La datation correspond à la fin Ier siècle apr. J.-C., en raison de la formule EX TESTAMENTO in extenso, de VIXIT ANNOS non abrégé, du formulaire initial au nominatif (sans mention aux dieux Mânes), du mot AERORVM pour indiquer les années de service, de I'installation de la legio VIII à Argentoratum (ca 90) et du contexte archéologique.

C. Taedius Secundus, le dédicataire, est un vétéran de la legio VIII a Augusta. Il reçoit cette stèle funéraire de C. Brinnius Quintus, vétéran, probablement de la même légion, son héritier.

[36] Les occurrences de ce nomen se situent exclusivement en Italie : A. Taedius, à Rome (CIL VI 29710) ; A. Taedius Fortunatus, à Rome (brique, CIL VI 1463) ; Taedia Secunda, à Pompéi (CIL IV 7469). Attesté aussi chez le grammairien Asconius, Commentaire sur les discours de Cicéron, III, «Sur le Pour Milon », 30, Sex. Taedius est un sénateur romain qui organise les funérailles de Clodius. S'agissant du nom Taedius dans notre inscription, il s'agit d'un unicum dans la cité de Vienne.
Le gentilice Taedius est italien [36]. En raison de son origo, la cité de Vienne, le défunt est un «Viennois hors de Vienne » (B. Rémy) [37], juridiquement rattaché à la civitas Viana Allobrogum. La forme Viana, au lieu de Vienna, n'est pas rare dans l'épigraphie [38].

La mort de Secundus, survenue à 50 ans, laisse présumer la date approximative de sa naissance, vers 40 apr. J.-C. à Vienne, à la fin du règne de Caligula. Ni sa tribu ni sa filiation patronymique ne sont indiquées, ce qui n'est pas conforme à l'état civil d'un citoyen romain. L'absence du lien agnatique filial pourrait laisser penser que son père, mort avant l'attribution du solidum ciuitatis Romanae beneficium par Caligula [39], n'était pas citoyen romain. Rien n'exclut que Secundus soit un citoyen de première génération.

Contrairement à C. Caprius Iulianus, C. Taedius Secundus est mort vétéran. On note qu'il a accompli vingt-cinq ans de service qui semblent la durée nécessaire, à l'époque flavienne, pour être qualifié de vétéran [40].

Sur la chronologie de la démobilisation de ce vétéran, on est réduit à des suppositions déduites de la date approximative du monument funéraire, de l'âge de décès du vétéran et enfin de la date hypothétique de son recrutement. Vingt-cinq ans durant, C. Taedius Secundus servit sous les enseignes de la légion VIII avant d'obtenir sa démobilisation par le diplôme de congé honorable en tant que vétéran. En admettant que son stipendium fut continu, il s'engagea à 25 ans. Comme G. Caprius Iulianus (cf supra), il dut être préalablement intégré dans le corps de troupe, installé à Mirebeau, destiné à réprimer la révolte des Lingons avant de suivre sa légion à Argentoratum au début des années 90 apr. J.-C.

D'après F. Tassaux, toutes périodes confondues, les Viennois arrivent en tête du recrutement des soldats gaulois dans l'armée romaine : avec 76 représentants (dont 69 issus de Vienne), ils ont davantage fourni de recrues (tirones) que les Voconces (22 recrues) et que les Volques Arécomiques (16 recrues) [41]. Le recrutement des Viennois dans les légions de Germanie supérieure fut très intense à en juger par le contingent des quatorze Allobroges officiant dans des légions stationnées sur le limes rhénan, dont dix à Mogontiacum/

[37] RÉMY 2005.

[38] Par exemple : CIL XIII 6871, 6872, 6944, 6972, 11859 et CSIR D 2, 5, 96 (Mogontiacum/Mayence) ; ILJug III 2818 (Dalmatie, Burnum/Ivosevci).

[39] ChRistol 2010, p. 23.

[40] Speidel 2009, p. 329 et Mann 2000, p. 155.

[41] TASSAUX 1996, p. 156. Voir aussi, sur le recrutement militaire des Viennois : FoRNi 1953, p. 180, appendice 2. 
Mayence [42]. Chez les Allobroges, une tradition forte de servir est enracinée, au point que des familles viennoises sont des « familles de militaires » dans lesquelles on sert de père en fils (Terentii, Lucconii). Toutefois, C. Taedius Secundus n'appartient pas à I'un de ces lignages qui servit dans les corps auxiliaires ou la légion.

Il est difficile d'établir les circonstances (volontariat ou conscription par dilectus) qui présidèrent à sa signatio, son inscription sur les rôles militaires. Son immatriculation dans la légion a pu être consécutive aux pillages des troupes du légat Vitellius, soutenues par les Lingons révoltés, qui s'en étaient rendu coupable jusqu'à Vienne [43]. Le rêve du centurionat pour les simples soldats (gregarii), ainsi que le montant annuel de la solde légionnaire, portée de 225 à 300 deniers par an en 83 apr. J.-C. par Domitien [44] n'y sont vraisemblablement pas étrangers.

B. Rémy a par ailleurs mis en évidence qu'à partir du début du II siècle, les Viennois ne s'engagent plus dans les légions romaines. Cette désaffection s'explique par le souci des autorités impériales de privilégier un recrutement local sur le limes rhénan (Ubiens, Bataves), mais aussi par les progrès de la « romanisation » et par la prospérité de la cité de Vienne [45]. C. Taedius Secundus appartient à cette dernière génération de Viennois qui s'engage massivement dans les légions.

Au terme de sa militia, C. Taedius Secundus accomplit les vingt-cinq ans réglementaires pour obtenir sa démobilisation de la légion. Lorsqu'il reçoit son certificat de congé honorable (honesta missio), lequel l'élève au rang de vétéran, il bénéficie aussi d'une prime de libération équivalant à 3000 deniers [46]. Cette missio nummaria - 12000 sesterces -, versée par le trésor militaire (aerarium militare), lui offre une retraite confortable. Son statut de vétéran range ce soldat démobilisé parmi les honestiores d'Argentoratum. Le titre d'honestior lui offre une immunité fiscale et lui confère une dignité égale à celle des décurions [47]. Nulle raison alors de s'étonner de sa capacité à financer une épitaphe monumentale.

Après sa démobilisation, Secundus ne rentra pas à Vienne dont il était pourtant originaire. Il fit le choix de rester près du camp où était stationnée sa garnison pour passer sa retraite près de ses anciens camarades légionnaires. Il s'intégra alors à la société de canabarii qui vivaient autour du camp. On ignore s'il reçut un lopin de terre, au titre de la missio agraria. Toutefois, en livrant les noms de deux nouveaux vétérans, cette inscription accroît les attestations à Argentoratum d'une présence de la huitième légion, jusque-là réduite épigraphiquement [48]. Elle ne préjuge donc nullement d'une colonisation du territoire et n'implique pas un lotissement collectif de terres.

C. Brinnus, le dédicant légataire, est l'exécuteur testamentaire. Secundus, le testateur, le coucha sur son testamentum avec l'ordre d'ériger son monumentum. On ignore l'origine de C. Brinnus, peut-être gauloise [49], mais son gentilice italien Brinn(i)us est très répandu en Campanie [50]. Pour C. Taedius Secundus, il s'agit d'un frère d'armes qui entretenait avec lui des liens de camaraderie militaire, d'esprit de corps et de compagnonnage. Dans ce contexte militaire, il y a une substitution de la «famille d'armes »- la «parenté de cœur» - à la parenté biologique.
[42] À Argentoratum/Strasbourg (Germanie Supérieure) : Q. Sertorius Quadratus, vétéran de la légion II ( $A E 2010$ 1067). A Mongontiacum/Mayence (Germanie Supérieure) : M. Petronius Mansuetus, miles dans légion IIII Macedonica (CIL XIII 6871) ; P. Solius Suavis, miles dans la légion XXII Primigenia (CIL XIII 6972) ; M. Sullius Campanus, miles dans la légion IIII Macedonica (CIL XIII 872) ; C. Dannius Secundus, miles dans la légion XIIII Gemina Martia Victrix (CIL XIII 6891); T. Tertius, miles dans la légion XIIII Gemina (CIL XIII 6909); T. Pompeius, miles dans la légion XVI (CIL XIII 6944); C. Helvius Sabinus, miles dans la légion IIII Macedonica (AE 1965 253) ; Sex. Valerius Mansuetus, miles dans la légion IIII Macedonica (CIL XIII 6873) ; L. Valerius Verecundus, miles dans la légion XIIII Gemina Martia Victrix (CIL XIII 6912) ; C. Rittius Paullus, miles dans la légion XXII Primigenia (CIL XIII 6969). A Vindonissa/ Windisch (Germanie Supérieure) : C. Valerius Campanus, miles dans la légion XI Claudia Pia Fidelis (CIL XIII 5214). À Wintersdorf (Germanie Supérieure) : (...)lius Perpetuus, miles dans la légion VIII Augusta (CIL XIII 6319). À Zurzach (Germanie Supérieure) : (...) Certus, vétéran de la légion XIII Gemina (CIL XIII 5239).

[43] REDDÉ 1996, p. 101.

[44] Suétone, Vie de Domitien, 7 ; Dion Cassius, Histoire romaine, LXVII, 3, 5.

[45] RÉMY 2005, p. 118.

[46] Dion Cassius, LIV, 25, 5-6.

[47] Digeste, XLIX, 18, 1 et 3.
[48] C. Celsinius Matutinus, vétéran (CIL XIII $11608=A E$ 2010 1067).

[49] En Gaule, Brennos, latinisé Brennus (variante allophonique du gaulois branos, le corbeau, avatar du dieu Lug), signifie littéralement, «chef, guerrier ». Ce substrat culturel gaulois a pu favoriser, par la consonance, le choix du gentilice Brinnus par le premier citoyen romain de sa famille. La forme in extenso de son surnom Quintus, qui contraste avec le praenomen, est attestée. Par exemple, le signifer L. Surius Quintus, à Trèves (AE 2000 981) ; le secrétaire P. Aelius Quintus à Delminium/Lib, en Dalmatie (AE 2003 1327) ; C. Amonius Quintus, à Wiesloch, en Germanie Supérieure (AE 1992 1282) ; le sonneur de cor Aelius Quintus à Aquicum/Budapest, en Pannonie Inférieure, Aurelius Quintus, à Rome (AE 1972 46).

[50] Brinnius : 16 occurrences (10 en Italie) : C. Brinnius Eutyches, à Rome (NSA 1923 375) ; L. Brinnius, à Rome (CIL VI 448); à Naples : Brinnia Helias, Brinnius Menander, Brinnia Parthenope, Brinnia Drosis (NSA 1892317 b) ; C. Brinnius Nicephorus, à Pouzzoles (CIL X 2175) ; Brinia Marciana à Pouzzoles (CIL X 1987) ; Brinnia Epigonia, à Pouzzoles (CIL $X$ 2176) ; Brinnia Procula à Iuuanum/Santa Maria di Palazzo, dans le Samnium (CIL IX 2969) ; L. Brinnius, à Lyon ( $A E$ 1996 1069) ; Brinnius Anthinus, à Valence (Hep. 1994 920), Brinnius Andronicus, à Valence (Hep. $1994932=A E 1991$ 1088) ; Brinnia Maxima, à Emona, en Pannonie Supérieure (ILJug. I 316) ; C. Brinnius Valentinus (ILAlg. II 2365) et Brinia Iucunda (ILAlg. II 2366), en Numidie. 


\section{SYNTHÈSE}

La découverte de ces deux stèles confirme la présence d'une nécropole destinée aux légionnaires et vétérans de la legio VIII ${ }^{a}$ Augusta à Koenigshoffen, à la fin du Irr siècle de notre ère. Ce pôle funéraire fait suite à celui du milieu du Ier siècle mis en place pour la legio II. La présence des restes d'un édicule, d'une sépulture secondaire à crémation, d'un bûcher funéraire en fosse, d'une fosse à dépôt d'offrandes, de dépôts céramiques et d'autres éléments lithiques en position secondaire [51], indique que la nécropole se poursuivait au moins sur la moitié ouest de la fouille du 2, route des Romains. Les réaménagements successifs depuis I'Antiquité jusqu'à nos jours ont malheureusement largement oblitéré les monuments funéraires.

L'étude générale du site indique bien que dès les années 120-140, des bâtiments furent construits dans la partie sud-ouest de la zone de fouille. La nécropole a été démantelée à cette période et les stèles enfouies pour laisser place aux nouveaux aménagements. Si elles ont été érigées à la fin du I Ir siècle, comme l'épigraphie l'atteste, elles ne sont restées en place qu'une trentaine d'années, ce qui semble un temps assez court. La pression démographique du vicus a certainement contraint les habitants à développer le territoire urbain vers l'est en oblitérant les sépultures. Lors du démantèlement de la nécropole, vu la masse de ces parallélépipèdes de grès, nous supposons qu'ils ont été enfouis sur place ou très proches de leur lieu d'érection. Même à l'époque contemporaine, Iors de leur redécouverte, ils ont probablement été peu déplacés. Le fait de les avoir trouvés dans la même fosse n'indique pas qu'ils aient été érigés I'un à côté de l'autre, ils ont pu être découverts au même moment à une certaine distance puis « jetés » au même endroit.

Les épitaphes de C. Caprius Iulianus, originaire de Toulouse, et $\mathrm{C}$. Taedius Secundus, originaire de Vienne, nous apportent des informations importantes sur les origines et l'histoire personnelle de ces deux légionnaires. Le type de matériau et l'ordonatio des inscriptions indiquent également une inégalité de statut entre eux : la somme léguée pour la réalisation de la stèle de l'architectus a certainement été plus importante, vu le travail soigné et la finesse du grès utilisé. Il semble bien que le traitement entre ces deux personnes ait été différent. Ces données enrichissent considérablement le corpus épigraphique de Strasbourg et permettent de mieux cerner l'importance de la légion VIII dans la région. .

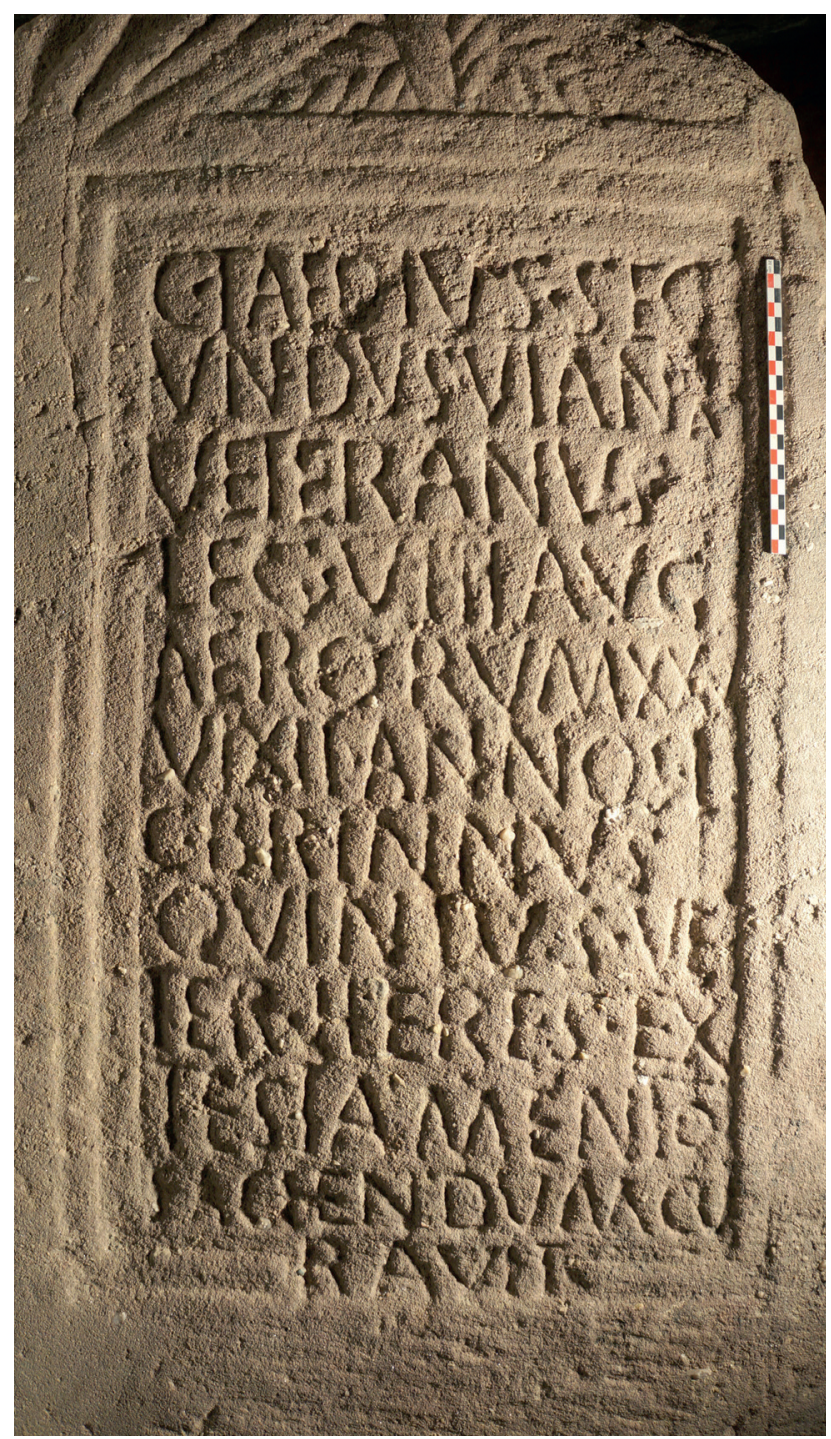

Fig. 11. Détail du titulus après nettoyage (Cliché $A$. Pichot)

[51] Pour un descriptif complet des structures liées à la nécropole de legio VIII ${ }^{a}$ voir le rapport de fouille MURER et al. 2020. 
Cet article est dédié à la mémoire de Bernard Rémy qui, au soir de sa vie, eut connaissance de C. Taedius Secundus, ce nouveau citoyen viennois, et relut cet article, gage ultime de sa curiosité pour la cité des Allobroges. Nos remerciements vont aussi aux experts pour leurs remarques et leurs conseils.

\section{BIBLIOGRAPHIE}

BARDouille, Jérôme, 2010, «L'importance du génie militaire dans l'armée romaine à l'époque impériale », Revue historique des armées 261, p. 79-87.

BLIN, Séverine, 2017, « Monuments funéraires de Koenigshoffen. Étude préliminaire des matériaux et types monumentaux 》, dans Bernadette Schnitzler \& Pascal Flotté (éd.), Koenigshoffen, époque romaine. Un quartier civil de Strasbourg-Argentorate du Ir-IVe siècle après J.-C., cat. Exp. Musée archéologique de Strasbourg, Strasbourg, p. 183-192.

BLIN, Séverine \& FLotrÉ, Pascal, 2017, « La nécropole de Strasbourg-Koenigshoffen du Irer au II siècle », dans Bernadette Schnitzler \& Pascal Flotté (éd.), Koenigshoffen, un quartier civil de Strasbourg-Argentorate du I ${ }^{\text {er }}$ au IV siècle ap. J.-C., Catalogue d'exposition du Musée archéologique de Strasbourg, Strasbourg, p. 174-180.

Christol, Michel, 2010, «L'organisation des communautés en Gaule méridionale (Transalpine, puis Narbonnaise) sous la domination de Rome », Pallas 84, p. 15-36.

Cosme, Pierre, 2012, L'année des quatre empereurs, Paris.

DaRdaIne, Sylvie, 2017, «De nouvelles inscriptions découvertes aux 8-20, Route des Romains en 2015 », dans Bernadette Schnitzler \& Pascal Flotté, Koenigshoffen, un quartier civil de Strasbourg-Argentorate du Ier au IVe siècle ap. J.-C., Catalogue de l'exposition au Musée archéologique de Strasbourg, Strasbourg, p. 180-182.

Donderer, Michael, 1996, Die Architekten der späten römischen Republik und der Kaiserzeit. Epigraphische Zeugnisse, Erlangen.

FAURE, Patrice, 2013, L'aigle et le cep. Les centurions légionnaires dans l'Empire des Sévères, Bordeaux.

Fleury, Philippe, 2011, «Vitruve et le métier d'ingénieur », Cahiers des études anciennes [En ligne], XLVIII.

FornI, Giovanni, 1953, Il reclutamento delle legioni da Augusto a Diocleziano, Roma.

ForNI, Giovanni, 1974, «Estrazione etnica e sociale dei soldati delle legioni nei primi tre secoli dell'Impero », dans Haase Temporini (éd.), Aufstieg und Niedergang der Römischen Welt (ANRW), II, 1, Berlin - New York, p. 339-391.

Goguey, René \& Redde, Michel, (éd.), 1995, Le camp légionnaire de Mirebeau, Mainz, (Monographien 36).

Goguey, René, 2008, «Légionnaires romains chez les Lingons : la VIII e Augusta à Mirebeau (Côte-d'Or) 》, Revue archéologique de l'Est 57, p. 227-251.

HARTKopf-Fröder, Christoph \& Jodry, Florent, 2016, « Comnisca, fils de Vedillus, Ambien, cavalier dans l'aile Indiana : étude pétrographique de l'exceptionnelle stèle funéraire découverte à Strasbourg », Revue archéologique de l'Est [En ligne], 65, mis en ligne le 10 octobre 2017.

Kajanto, Iiro, 1965, The Latin cognomina, Helsinki.

KolB, Anne, 2008, « Das Bauhandwerk in den Städten der römischen Provinzen. Strukturen und Bedeutung », Tyche 23, p. 101-115.

KunnLe, Gertrud, 2018, Argentorate : le camp de la VIII légion et la présence militaire romaine à Strasbourg, Mainz (Monographien des Römisch-Germanischen Zentralmuseums 141).

LöRINCz, Barnabas, 1999, Onomasticon provinciarum Europae latinarum (OPEL), Wien.

MANN, John Cecil, 1983, Legionary recruitment and veteran settlement during the Principate, London.

ManN, John Cecil, 2000, « Honesta missio from the legions», dans Géza Alföldy, Brian Dobson \& Werner Eck (éd.), Kaiser, Heer und Gesellschaft in der römischen Kaiserzeit: Gedenkschrift für Eric Birley, Stuttgart, p. 153-161.

Murer, Axelle, Barrand-Émam, Hélène, Beraud, Marianne, Bonte, Marie-Laure, Broc, Émeline, Collombet, Julien, Daverat, Loïc, Fabre, Magali, Gohier, Pauline, Goudissard, Simon, Pichot, Adeline, Schott-Toullec, Laetitia, 
Tenud, Antoine, Touzet, Amandine \& Viero, Anne, 2020, Strasbourg-Koenigshoffen, Extension du Tramway - 2, route des Romains, Alsace, Bas-Rhin (67), Rapport final d'opération d'archéologie préventive, SRA Grand Est, Habsheim. PaIller, Jean-Marie, 1988, « Domitien et la "Cité de Pallas", un tournant dans I'histoire de Toulouse antique », Pallas 34, p. 99-109.

Provost, Michel \& Pailler, Jean-Marie, 2017, Toulouse 31-3, Paris (Carte Archéologie de la Gaule 31/3).

Redde, Michel, 1993, « Le camp de Mirebeau et l'histoire de la VIII légion Auguste sous les Flaviens », dans Yann Le Bohec (éd.), Militaires romains en Gaule civile, Actes de la table ronde de Lyon (mai 1991), Paris, p. 45-50.

RedDe, Michel, 1996a, « Le camp légionnaire de Mirebeau », dans Michel Reddé (dir.), L'armée romaine en Gaule, Paris, p. 191-202.

RedDe, Michel, 1996b, « Le camp de Strasbourg », dans Michel Reddé (dir.), L'armée romaine en Gaule, Paris, p. $203-207$.

RedDe, Michel, 1997, «Réflexions sur l'occupation militaire de Strasbourg et de Mirebeau au Ier siècle apr. J.-C. », Gesellschaft Pro Vindonissa, p. 5-12.

RedDe, Michel, 2000, « La legio VIII Augusta », dans Yann Le Bohec \& Catherine Wolff (éd.), Les légions de Rome sous le Haut-Empire, Lyon, p. 122-126.

Redde, Michel, 2006, «Mirebeau-sur-Bèze. Le camp de la VIIIe légion », dans Pierre Aupert, Michel Reddé, Raymond Brulet \& Rudolf Fellmann (éd.), L'architecture de la Gaule romaine. Les fortifications militaires, Bordeaux (Documents d'archéologie française 100), p. 331-333.

RedDE, Michel, 2010, « La garnison de Strasbourg et la conquête de la Germanie », dans Bernadette Schnitzler et Gertrud Kuhnle (dir.), Strasbourg-Argentorate : un camp légionnaire sur le Rhin (I er au IV siècle après J.-C.), Catalogue d'exposition du Musée archéologique de Strasbourg, Strasbourg, p. 29-32.

Remy, Bernard, 2005, Les Viennois hors de Vienne : attestations (épigraphiques, littéraires et papyrologiques) de l'activité des Viennois(es) en dehors de leur cité, Bordeaux (Mémoire - Ausonius 15).

Schmidt Heidenreich, Christophe, 2012, « Les soldats bâtisseurs dans les camps sous le Haut-Empire », dans Catherine Wolff (dir.), Le métier de soldat dans le monde romain, Actes du cinquième congrès de Lyon, 23-25 septembre 2010, Paris, p. 327-344.

Schnitzler, Bernadette \& Kunnle, Gertrud (dir.), 2010, Strasbourg-Argentorate : un camp légionnaire sur le Rhin

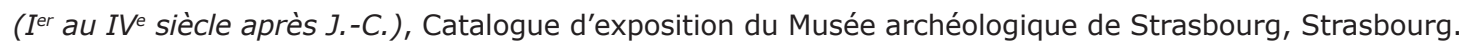

Sperdel, Michael Alexander, 2009, « Honesta missio. Zu Entlassungsurkunden und verwandten Texten », dans Michael Alexander Speidel (éd.), Heer und Herrschaft im römischen Reich der hohen Kaiserzeit, Stuttgart, p. 317-346.

SToLl, Oliver, 1998, « Ordinatus Architectus. Römische Militärarchitekten und ihre Bedeutung für den Technologietransfer », dans Leonhard Schumacher (éd.), Religion - Wirtschaft - Technik. Althistorische Beiträge zur Entstehung neuer kultureller Strukturmuster im historischen Raum; allgemein Nordafrika / Kleinasien / Syrien, St. Katarinen, p. $203-271$.

Tassaux, Danielle \& Tassaux, Francis, 1996, « Les soldats gaulois dans l'armée romaine », dans Michel Reddé (dir.), L'armée romaine en Gaule, Paris, p. 147-164. 